\title{
Demographic profile of patients treated for melasma with glycolic acid and trichloroacetic acid: an institutional experience
}

\author{
Bikkasani U. P. Lakshmi Kishan Rao*, K. Satyanarayana Rao
}

Department of DVL, Mamata Medical College, Khammam, Telangana, India

Received: 15 June 2021

Accepted: 02 July 2021

\author{
*Correspondence: \\ Dr. Bikkasani U. P. Lakshmi Kishan Rao, \\ E-mail: mamatakhmm@gmail.com
}

Copyright: ( $)$ the author(s), publisher and licensee Medip Academy. This is an open-access article distributed under the terms of the Creative Commons Attribution Non-Commercial License, which permits unrestricted non-commercial use, distribution, and reproduction in any medium, provided the original work is properly cited.

\begin{abstract}
Background: Melasma is an acquired disorder of hyperpigmentation characterised by forehead, cheeks, around eyelids and chin mostly in the sun-exposed areas of the skin. The etiology for melasma is not clear, various factors like genetic or hormonal influences the cause. The aim was to understand the demographical distribution and epidemiological pattern of pigmentation in melasma patients.

Methods: A total of 50 patients were participated in the present study at department of dermatology of Mamata medical college and hospital, Khammam over a period of one year.

Results: The mean age of patients with melasma was 33.84 \pm 5.76 years, with the youngest and oldest being 20 and 40 years with female preponderance, with a male to female ratio of 1:9. The maximum number of patients educated up to middle school level $(38 \%)$ followed by primary school. The maximum number of individuals participated in the present study were housewife's and out of 50 individuals $92 \%$ patients in the present series were married whereas family history of melasma was noted only $16 \%$ of the melasma patients.

Conclusions: The present observations demonstrates that the melasma is a female predominant, affecting young adults, etiological observations like sunlight, married, pregnancy, indoor, family history followed by cosmetics plays an important role in the melasma.
\end{abstract}

Keywords: Melasma, Demographical profile, MASI scoring, Glycolic acid and trichloroacetic acid

\section{INTRODUCTION}

Melasma condition is more commonly seen in women with dark complexion of Fitzpatrick skin types (IV and V) especially those of Hispanic, African American, Arab, Southeast Asian and East Asian descent. Melasma accounts for 0.25 to $4 \%$ of the patients seen in dermatology clinics in South East Asia and is the most common pigment disorder among Indians. ${ }^{1}$

Multiple etiologic factors have been implicated like high estrogen states (pregnancy, oral contraceptives), genetic factors, cosmetics and autoimmune thyroid disease. Sunlight exposure appears to be essential for its development. $^{2}$ Apart from the above factors the exact mechanism of etiopathogenesis of melasma is still unclear. Some earlier authors have suggested that excess melanocytosis and melanogenesis and also increase in vascular endothelial growth factor were also responsible for the hyperpigmentation in melasma. ${ }^{3}$

The hyperpigmented patches may range from single to multiple, usually symmetrical on the face and occasionally V-neck area. According to the distribution of lesions, three clinical patterns of melasma are recognized. The centrofacial pattern is the most common pattern and involves the forehead, cheeks, upper lip, nose and chin. The malar pattern involves the cheeks and nose. The mandibular pattern involves the ramus of the mandible. 
Various combination of topical medications like hydroquinone, tretinoin, topical steroids are also used for melasma but chemical peeling provides more rapid response to topical therapy. ${ }^{4}$ Chemical peeling is a popular, relatively inexpensive and generally safe method for the treatment of cutaneous pigmentation. ${ }^{5}$ There is a wide array of peeling agents available in the market today, with different formulations and combinations. Those most commonly used include phenol, trichloroacetic acid (TCA), alpha hydroxyacids (AHAs), and beta hydroxyacids. It has been established that chemical peels potentiate the effect of the depigmenting agents and reduce significantly the melasma area and severity index (MASI). ${ }^{6}$

Glycolic acid (GA) and TCA chemical peels have been used along and in combination to treat melasma, however no studies have been carried out on this subject from this part of the country, as far as ascertained. ${ }^{7}$ Despite melasma is one of the most common skin problems encountered. Hence the present study was aimed to study the demographical distribution and epidemiological pattern of pigmentation in melasma patients.

\section{METHODS}

Our study was in a prospective observational category study. This study was approved by the institutional scientific and ethical committee review board. Fifty patients with clinically diagnosed melasma of either sex attending the outpatient clinic of department of dermatology of Mamata medical college and hospital, Khammam during the period of April 2007 to March 2008 constituted the subject material.

\section{Inclusion criteria}

Patients with clinically diagnosed melasma and patients willing to undergo the procedure of chemical peels by giving an informed consent were included in the study.

\section{Exclusion criteria}

Pregnant and nursing women, patients with a history of recurrent herpes labialis, patients with hypersensitivity to any of the formulations (GA, retinoic acid, hydroquinone) were excluded from the study.

\section{Methods}

Detailed history regarding age, sex, occupation and education of patients, nature and duration of complaints, age of onset, site of onset of lesions, precipitating and exacerbating factors and family history were recorded in a prefabricated proforma.

A detailed dermatological examination was carried out and the morphology, extent and anatomical location of the lesions were recorded. Based on examination under natural light, the lesions were classified as light brown, medium brown, dark and bluish-grey in color. A further division into epidermal or mixed was made using Wood's lamp.

In all patients, skin typing was done according to Fitzpatrik's classification. An informed consent was taken from all the patients before the procedure after explaining the benefits, side-effects and risks associated with the procedure.

All the study participants were randomly assigned in 2 groups (A and B). Group A and B consisting of 25 patients each. Group A had GA peels only. Group B had TCA peels only.

Each group colored photographs of the patient were taken before each peel and severity was assessed by MASI scoring.

\section{Statistical analysis}

Statistical analysis of the results was done by calculation the average MASI scores for the 2 groups and evaluation the percentage change in the average MASI scores wad done before each peel and thirty days after last peel of treatment using unpaired t test and one way ANOVA tests. A $p$ value of less than 0.05 was considered significant.

\section{RESULTS}

The data observed in compelled in tabled forms and therapeutic benefit has been documented.

The youngest patients with melasma were 20 years old while oldest was 40 years with a mean age of $33.84 \pm 5.76$. Statistical analysis revealed that the two groups were comparable with respect to the age incidence (Table 1).

In the present study, maximum number of patients educated up to middle school level $(38 \%)$ followed by primary school (30\%) (Table 2).

The present study shows that in group A, 23 (92\%) cases were females and only $2(8 \%)$ males. Similarly, in group B, $22(88 \%)$ cases were females and only $3(12 \%)$ males as shown in Figure 1. Amongst the 50 cases included in the study, $45(90 \%)$ were females and $5(10 \%)$ were males, with a male to female ratio of $1: 9$. The sex incidence in the two groups did not reveal any statistically significant difference.

Housewife $(52 \%)$ constituted the single largest group followed by daily laborers $(20 \%)$, teachers and staff nurses $(8 \%)$, social workers $(4 \%)$, advocate $(2 \%)$ and tailor $(2 \%)$ (Table 3$)$.

In the present study, out of 50 individuals $92 \%$ patients in the present series were married (Figure 2). 
Table 1: Age distribution of patients with melasma.

\begin{tabular}{|lll|}
\hline Age group (in years) & $\begin{array}{l}\text { Group A } \\
(\mathbf{n}=\mathbf{2 5})(\%)\end{array}$ & $\begin{array}{l}\text { Group B } \\
(\mathbf{n}=\mathbf{2 5})(\%)\end{array}$ \\
\hline $\mathbf{2 0 - 3 0}$ & $9(36)$ & $15(60)$ \\
\hline $\mathbf{3 1 - 4 0}$ & $12(48)$ & $10(40)$ \\
\hline $\mathbf{> 4 0}$ & $4(16)$ & $0(0)$ \\
\hline Total & 25 & 25 \\
\hline Mean \pm SD & $33.84 \pm 7.28$ & $30.32 \pm 5.76$ \\
\hline
\end{tabular}

$\mathrm{T}$ value $=1.90, \mathrm{p}>0.05 \mathrm{~N} \mathrm{~S}$.

Table 2. Educational status of patients with melasma.

\begin{tabular}{|lll|}
\hline Education & Group A and B & Percentage \\
\hline Illiterate & 5 & 10 \\
\hline Primary school & 15 & 30 \\
\hline Middle school & 19 & 38 \\
\hline Secondary school & 5 & 10 \\
\hline Graduate & 6 & 12 \\
\hline Total & 50 & 100 \\
\hline
\end{tabular}

Table 3: Distribution of individuals according to occupational status of patients of melasma.

\begin{tabular}{|lll|}
\hline Occupation & Group A and B & Percentage \\
\hline Housewife & 26 & 52 \\
\hline Teacher & 4 & 8 \\
\hline Staff nurse & 4 & 8 \\
\hline Daily labor & 10 & 20 \\
\hline Security guard & 2 & 4 \\
\hline Advocate & 1 & 2 \\
\hline Tailor & 1 & 2 \\
\hline Social worker & 2 & 4 \\
\hline Total & 50 & 100 \\
\hline
\end{tabular}

Table 4. Distribution of melasma patients by lifestyle.

\begin{tabular}{|lll|}
\hline Lifestyle & Group A and B & Percentage \\
\hline Indoors & 38 & 76 \\
\hline Outdoors & 12 & 24 \\
\hline Total & 50 & 100 \\
\hline
\end{tabular}

Table 5. Distribution of melasma patients by family history.

\begin{tabular}{|lll|}
\hline Family history & Group A and B & Percentage \\
\hline Present & 8 & 16 \\
\hline Absent & 42 & 84 \\
\hline Total & 50 & 100 \\
\hline
\end{tabular}

Majority of the patients had a lifestyle in which they stayed indoors most of the time while $24 \%$ were involved more often in outdoor activities (Table 4).

In the present study, family history of melasma was noted only $16 \%$ of the melasma patients (Table 5 ).

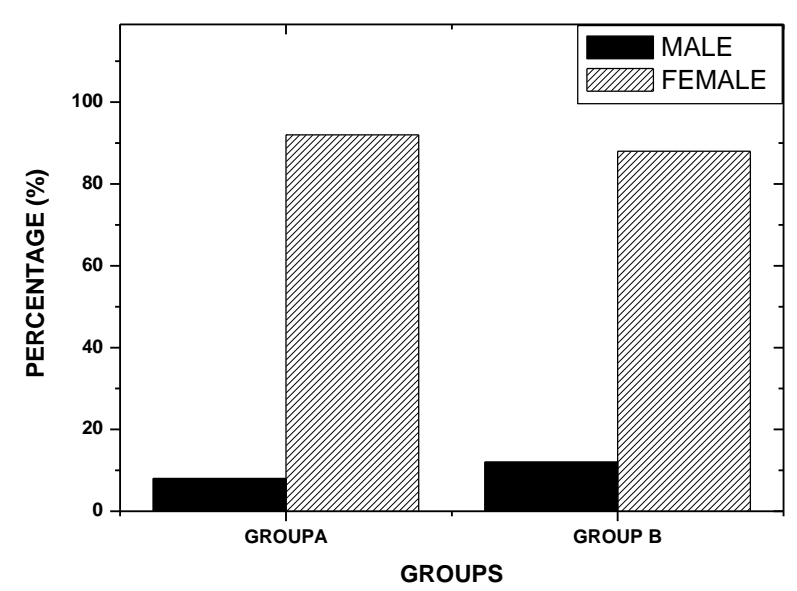

Figure 1: Sex distribution of patients with melasma in group A and group B.

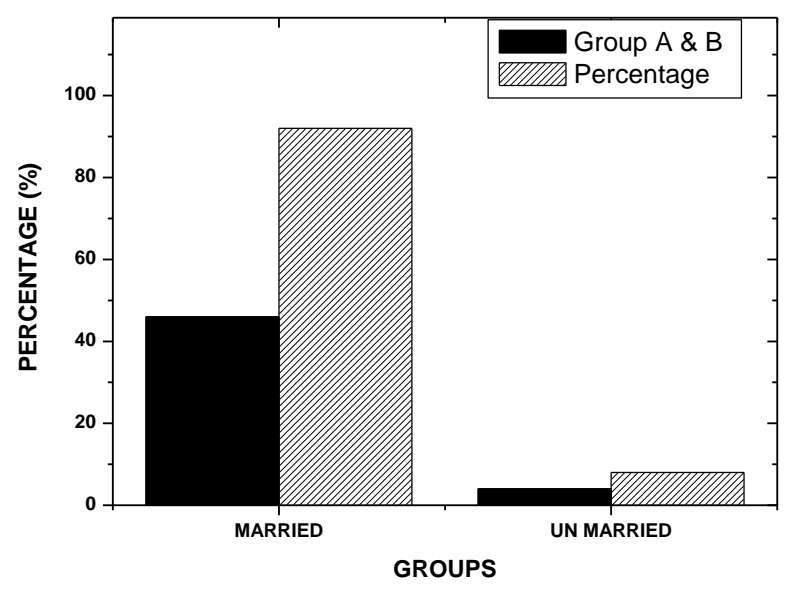

Figure 2: Distribution of individuals according to marital status of patients with melasma.

\section{DISCUSSION}

Melasma is a common disorder of acquired hyperpigmentation characterized by tan or brown macules and patches localized to photo-exposed areas of the face, particularly the malar areas, forehead and chin. ${ }^{8}$ Various factors implicated in occurrence of melasma includes genetic predisposition, ultraviolet light exposure, pregnancy, oral contraceptives, hormone replacement therapy, thyroid disease, cosmetics and medications. ${ }^{9}$ Melasma, one of the common aesthetically displeasing entities, continues to be a difficult problem to treat. Different topical depigmenting agents have been used alone or in combination with varying results but no agent have proved to be ideal. Chemical peeling is one new weapon in the therapeutic armamentarium of melasma. GA and TCA chemical peels have been used alone and also in combination to treat melasma. ${ }^{10}$

In the present study we aimed to study the demographical distribution and epidemiological pattern of pigmentation in melasma. Patients were assigned randomly two groups, 
group A and group B. Patients in group A underwent chemical peeling with GA and patients in group B with TCA. There peels were carried out at three weekly intervals. Patients were followed up for a period 30 days after the last peel. The response in each group was observed.

The means age of patients in group A was 33.84 years and in group B were 30.326 years. The cases included in the study varied in age from 20-46 years. Out of the total 50 cases, $92 \%$ were in the age group of 20-40 years. In group A, $84 \%$ cases were in the age group of 20-40 years whereas, in group B $100 \%$ cases fell in this age group. Our present results are in accordance with earlier recent study conducted by Achar and co-workers in 312 Indian patients, the mean age of patients with melasma was 33.45 years, ranging from 14 to 54 years. There was a female preponderance with a female-to-male ratio of approximately $4: 1$. The mean age of onset was 29.99 years, with the youngest and oldest being 11 and 49 years, respectively. ${ }^{11}$

Similar observations were also made by Kalla et al reported melasma to be more prevalent in the younger age group, ( $87 \%$ between $20-40$ years). In their study the age range varied from 17-45 years. ${ }^{12}$ Whereas Javaheri et al in their study reported maximum incidence of melasma in the age group of 24-45 years. ${ }^{13}$ Thus the present study corroborates with the findings in the previous two studies regarding the age incidence.

In the present study, out of the total 50 patients, 45 cases $90 \%$ were females and $5(10 \%)$ males, with a male to female ratio of 1:9. In group A, $92 \%$ cases were females and only $8 \%$ were males. Similarly in group B, $88 \%$ cases were females and only $12 \%$ males. Thus, in both the groups, there was a female preponderance. In a similar study conducted by Kalla et al it was observed that melasma included in their study, 39\% were males and $61 \%$ females. ${ }^{10}$ Also in study by Sarkar et al out of 40 melasma patients, 22 were females and 18 males, showing female predominance. ${ }^{14}$ Javaheri et al conducted their study on melasma only in female patients. ${ }^{13}$

Our observations show that, maximum number of patients were educated up to middle school level $(38 \%)$ followed by primary school $(30 \%)$. These findings match with Katsambas et al. ${ }^{15}$ Present study was also done on distribution of individuals according to occupational status of patients of melasma, the results clearly state that, housewife constituted the single largest group followed by daily laborers, teachers and staff nurses, social workers, advocate and tailor such observations are also shared by other earlier studies. ${ }^{16}$

In our study $92 \%$ patients were married and as married women had more risk factors like pregnancy and use of OC pills specially estrogen containing or combine pills can trigger melasma which was evident from earlier study. Present study also infers that, majority of the patients had a lifestyle in which they stayed indoors most of the time while only $24 \%$ were involved in outdoor activities with a family history noted only in $16 \%$ of the melasma patients such observations are in accordance with earlier studies. ${ }^{16}$

\section{CONCLUSION}

To conclude from the present observations that the melasma is a female predominant, affecting young adults, etiological observations like sunlight, married, pregnancy, indoor, family history followed by cosmetics plays an important role and contributes in the melasma. Still more studies need to be performed to understand and to identify the causative factors and provocation to develop melasma.

\section{Funding: No funding sources}

Conflict of interest: None declared

Ethical approval: The study was approved by the institutional ethics committee

\section{REFERENCES}

1. Sarkar R, Arora P, Garg VK, Sonthalia S, Gokhale N. Melasma update. Indian Dermatol Online J. 2014;5(4):426-35.

2. Grimes PE. Melasma: etiologic and therapeutic consideration. Arch Dermatol. 1995;131(12):1453-7.

3. Lapeere H, Borne B, Schepper S, Verhaeghe E, Ongenae K, Geel NV. Hypomelanosis and hypermelanosis. In: Wolff K, Goldsmith LA, Katz SI, Gilchrest BA, Paller AS, Leffell DJ, eds. Dermatology in General Medicine. 7th ed. New York: McGraw-Hill; 2008: 622-40.

4. Yu RJ, Scott EJV. Bioavailability of alpha hydroxyl acids in topical formulations. Cosmetic Dermatol. 1996;9:54-6.

5. Brody H. History and classification of chemical peels. In: Patterson AN, eds. Chemical peeling. 1st ed. St. Louis: Mosby Book Inc; 1992: 7-22.

6. Finlay AY. 1997. Quality of life measurement in dermatology: a practical guide. $\mathrm{Br} \mathrm{J}$ Dermatol. 1997;136(3):305-14.

7. Coleman WP, Futrell JM. The glycolic acid trichloroacetic acid peel. J Dermatol Surg Oncol. 1994;20(1):76-80.

8. Sanchez NP, Pathak MA, Sato S, Fitxpatrick TB, Snachez JL, Mihm MC. Melasma: A clinical, light microscopic, ultra-structural, and immunofluorescence study. J Am Acad Dermatol. 1981;4(6):698-710.

9. Patel NH, Gangaiah N, Thimappa V, Gundappa P. Clinico-epidemiological study of melasma in tertiary care centre. Int J Res Dermatol. 2020Jul;6(4):544-7.

10. Manjunath KG, Raghu MT, Yogendra M, Harish G. A clinical and therapeutic study of efficacy of $40 \%$ 
glycolic acid facial peels in melasma. Int $\mathrm{J}$ Res Dermatol. 2018;4(2):136-41.

11. Achar A, Rathi SK. Melasma: a clinicepidemiological study of 312 cases. Indian J Dermatol. 2011;56(4):380-2.

12. Kalla G, Garg A, Kachhawa D. Chemical peeling: glycolic acid versus Trichloroacetic acid in melasma. Indian J Dermatol Venereol Leprol. 2001;67(2):82-4.

13. Javaheri SM, Handa S, Kaur I, Kumar B. Safety and efficacy of glycolic acid facial peel in Indian women with melasma. Int J Dermatol. 2001;40(5):354-7.

14. Sarkar R, Kaur C, Bhalla M, Kanwar AJ. The combination of glycolic acid peels with a topical regimen the treatment of Melasma in Dark skinned patients: a comparative study. Dermatol Surg. 2002;28(9):828-32.

15. Katsambas A, Soura E. Quality of life in melasma. Melasma and vitiligo in brown skin. 1st ed. New Delhi: Springer; 2017: 169-75.

16. Arora P, Meena N, Sharma PK, Raihan M. 2017. Impact of melasma on quality of life in Indian patients. Pigment Int. 2017;4(2):92.

Cite this article as: Rao BUPLK, Rao KS.

Demographic profile of patients treated for melasma with glycolic acid and trichloroacetic acid: an institutional experience. Int J Res Dermatol 2021;7:687-91. 